\title{
Affine Independence in Vector Spaces
}

\author{
Karol Pąk \\ Institute of Informatics \\ University of Białystok \\ Poland
}

Summary. In this article we describe the notion of affinely independent subset of a real linear space. First we prove selected theorems concerning operations on linear combinations. Then we introduce affine independence and prove the equivalence of various definitions of this notion. We also introduce the notion of the affine hull, i.e. a subset generated by a set of vectors which is an intersection of all affine sets including the given set. Finally, we introduce and prove selected properties of the barycentric coordinates.

MML identifier: RLAFFIN1, version: $\underline{7.11 .05 \quad 4.134 .1080}$

The terminology and notation used here are introduced in the following papers: [1], [6], [10], [2], [3], [8], [15], [13], [12], [11], [7], [5], [9], [14], and [4].

\section{Preliminaries}

For simplicity, we adopt the following convention: $x, y$ are sets, $r, s$ are real numbers, $S$ is a non empty additive loop structure, $L_{1}, L_{2}, L_{3}$ are linear combinations of $S, G$ is an Abelian add-associative right zeroed right complementable non empty additive loop structure, $L_{4}, L_{5}, L_{6}$ are linear combinations of $G$, $g, h$ are elements of $G, R_{1}$ is a non empty RLS structure, $R$ is a real linear space-like non empty RLS structure, $A_{1}$ is a subset of $R, L_{7}, L_{8}, L_{9}$ are linear combinations of $R, V$ is a real linear space, $v, v_{1}, v_{2}, w, p$ are vectors of $V, A, B$ are subsets of $V, F_{1}, F_{2}$ are families of subsets of $V$, and $L, L_{10}, L_{11}$ are linear combinations of $V$.

Let us consider $R_{1}$ and let $A$ be an empty subset of $R_{1}$. Note that conv $A$ is empty. 
Let us consider $R_{1}$ and let $A$ be a non empty subset of $R_{1}$. One can check that $\operatorname{conv} A$ is non empty.

One can prove the following propositions:

(1) For every element $v$ of $R$ holds $\operatorname{conv}\{v\}=\{v\}$.

(2) For every subset $A$ of $R_{1}$ holds $A \subseteq \operatorname{conv} A$.

(3) For all subsets $A, B$ of $R_{1}$ such that $A \subseteq B$ holds conv $A \subseteq \operatorname{conv} B$.

(4) For all subsets $S, A$ of $R_{1}$ such that $A \subseteq \operatorname{conv} S$ holds conv $S=$ conv $S \cup A$.

(5) Let $V$ be an add-associative non empty additive loop structure, $A$ be a subset of $V$, and $v, w$ be elements of $V$. Then $(v+w)+A=v+(w+A)$.

(6) For every Abelian right zeroed non empty additive loop structure $V$ and for every subset $A$ of $V$ holds $0_{V}+A=A$.

(7) For every subset $A$ of $G$ holds $\operatorname{Card} A=\operatorname{Card}(g+A)$.

(8) For every element $v$ of $S$ holds $v+\emptyset_{S}=\emptyset_{S}$.

(9) For all subsets $A, B$ of $R_{1}$ such that $A \subseteq B$ holds $r \cdot A \subseteq r \cdot B$.

(10) $(r \cdot s) \cdot A_{1}=r \cdot\left(s \cdot A_{1}\right)$.

(11) $1 \cdot A_{1}=A_{1}$.

(12) $0 \cdot A \subseteq\left\{0_{V}\right\}$.

(13) For every finite sequence $F$ of elements of $S$ holds $\left(L_{2}+L_{3}\right) \cdot F=$ $L_{2} \cdot F+L_{3} \cdot F$.

(14) For every finite sequence $F$ of elements of $V$ holds $(r \cdot L) \cdot F=r \cdot(L \cdot F)$.

(15) Suppose $A$ is linearly independent and $A \subseteq B$ and $\operatorname{Lin}(B)=V$. Then there exists a linearly independent subset $I$ of $V$ such that $A \subseteq I \subseteq B$ and $\operatorname{Lin}(I)=V$.

\section{Two Transformations of Linear Combinations}

Let us consider $G, L_{4}, g$. The functor $g+L_{4}$ yielding a linear combination of $G$ is defined as follows:

(Def. 1) $\left(g+L_{4}\right)(h)=L_{4}(h-g)$.

Next we state several propositions:

(16) The support of $g+L_{4}=g+$ the support of $L_{4}$.

(17) $g+\left(L_{5}+L_{6}\right)=\left(g+L_{5}\right)+\left(g+L_{6}\right)$.

(18) $v+r \cdot L=r \cdot(v+L)$.

(19) $g+\left(h+L_{4}\right)=(g+h)+L_{4}$.

(20) $g+\mathbf{0}_{\mathrm{LC}_{G}}=\mathbf{0}_{\mathrm{LC}_{G}}$.

(21) $0_{G}+L_{4}=L_{4}$. 
Let us consider $R, L_{7}, r$. The functor $r \circ L_{7}$ yields a linear combination of $R$ and is defined as follows:

(Def. 2)(i) For every element $v$ of $R$ holds $\left(r \circ L_{7}\right)(v)=L_{7}\left(r^{-1} \cdot v\right)$ if $r \neq 0$,

(ii) $r \circ L_{7}=\mathbf{0}_{\mathrm{LC}_{R}}$, otherwise.

The following propositions are true:

(22) The support of $r \circ L_{7} \subseteq r \cdot\left(\right.$ the support of $L_{7}$ ).

(23) If $r \neq 0$, then the support of $r \circ L_{7}=r \cdot\left(\right.$ the support of $\left.L_{7}\right)$.

(24) $r \circ\left(L_{8}+L_{9}\right)=r \circ L_{8}+r \circ L_{9}$.

(25) $r \cdot(s \circ L)=s \circ(r \cdot L)$.

(26) $r \circ \mathbf{0}_{\mathrm{LC}_{R}}=\mathbf{0}_{\mathrm{LC}_{R}}$.

(27) $r \circ\left(s \circ L_{7}\right)=(r \cdot s) \circ L_{7}$.

(28) $1 \circ L_{7}=L_{7}$.

\section{The Sum of Coefficients of a Linear Combination}

Let us consider $S, L_{1}$. The functor sum $L_{1}$ yields a real number and is defined as follows:

(Def. 3) There exists a finite sequence $F$ of elements of $S$ such that $F$ is one-toone and $\operatorname{rng} F=$ the support of $L_{1}$ and $\operatorname{sum} L_{1}=\sum\left(L_{1} \cdot F\right)$.

One can prove the following propositions:

(29) For every finite sequence $F$ of elements of $S$ such that the support of $L_{1}$ misses $\operatorname{rng} F$ holds $\sum\left(L_{1} \cdot F\right)=0$.

(30) Let $F$ be a finite sequence of elements of $S$. If $F$ is one-to-one and the support of $L_{1} \subseteq \operatorname{rng} F$, then $\operatorname{sum} L_{1}=\sum\left(L_{1} \cdot F\right)$.

(31) $\operatorname{sum} \mathbf{0}_{\mathrm{LC}_{S}}=0$.

(32) For every element $v$ of $S$ such that the support of $L_{1} \subseteq\{v\}$ holds $\operatorname{sum} L_{1}=L_{1}(v)$.

(33) For all elements $v_{1}, v_{2}$ of $S$ such that the support of $L_{1} \subseteq\left\{v_{1}, v_{2}\right\}$ and $v_{1} \neq v_{2}$ holds $\operatorname{sum} L_{1}=L_{1}\left(v_{1}\right)+L_{1}\left(v_{2}\right)$.

(34) $\operatorname{sum} L_{2}+L_{3}=\operatorname{sum} L_{2}+\operatorname{sum} L_{3}$.

(35) $\operatorname{sum} r \cdot L=r \cdot \operatorname{sum} L$.

(36) $\operatorname{sum} L_{10}-L_{11}=\operatorname{sum} L_{10}-\operatorname{sum} L_{11}$.

(37) $\operatorname{sum} L_{4}=\operatorname{sum} g+L_{4}$.

(38) If $r \neq 0$, then $\operatorname{sum} L_{7}=\operatorname{sum} r \circ L_{7}$.

(39) $\sum(v+L)=\operatorname{sum} L \cdot v+\sum L$.

(40) $\sum(r \circ L)=r \cdot \sum L$. 


\section{Affine Independence of Vectors}

Let us consider $V, A$. We say that $A$ is affinely independent if and only if:

(Def. 4) $\quad A$ is empty or there exists $v$ such that $v \in A$ and $(-v+A) \backslash\left\{0_{V}\right\}$ is linearly independent.

Let us consider $V$. Observe that every subset of $V$ which is empty is also affinely independent. Let us consider $v$. One can check that $\{v\}$ is affinely independent. Let us consider $w$. Observe that $\{v, w\}$ is affinely independent.

Let us consider $V$. Note that there exists a subset of $V$ which is non empty, trivial, and affinely independent.

We now state three propositions:

(41) $A$ is affinely independent iff for every $v$ such that $v \in A$ holds $(-v+A) \backslash$ $\left\{0_{V}\right\}$ is linearly independent.

(42) $A$ is affinely independent if and only if for every linear combination $L$ of $A$ such that $\sum L=0_{V}$ and $\operatorname{sum} L=0$ holds the support of $L=\emptyset$.

(43) If $A$ is affinely independent and $B \subseteq A$, then $B$ is affinely independent.

Let us consider $V$. Note that every subset of $V$ which is linearly independent is also affinely independent.

In the sequel $I$ denotes an affinely independent subset of $V$.

Let us consider $V, I, v$. Observe that $v+I$ is affinely independent.

One can prove the following proposition

(44) If $v+A$ is affinely independent, then $A$ is affinely independent.

Let us consider $V, I, r$. One can check that $r \cdot I$ is affinely independent.

The following propositions are true:

(45) If $r \cdot A$ is affinely independent and $r \neq 0$, then $A$ is affinely independent.

(46) If $0_{V} \in A$, then $A$ is affinely independent iff $A \backslash\left\{0_{V}\right\}$ is linearly independent.

Let us consider $V$ and let $F$ be a family of subsets of $V$. We say that $F$ is affinely independent if and only if:

(Def. 5) If $A \in F$, then $A$ is affinely independent.

Let us consider $V$. Observe that every family of subsets of $V$ which is empty is also affinely independent. Let us consider $I$. One can check that $\{I\}$ is affinely independent.

Let us consider $V$. Note that there exists a family of subsets of $V$ which is empty and affinely independent and there exists a family of subsets of $V$ which is non empty and affinely independent.

Next we state two propositions:

(47) If $F_{1}$ is affinely independent and $F_{2}$ is affinely independent, then $F_{1} \cup F_{2}$ is affinely independent.

(48) If $F_{1} \subseteq F_{2}$ and $F_{2}$ is affinely independent, then $F_{1}$ is affinely independent. 


\section{Affine Hull}

Let us consider $R_{1}$ and let $A$ be a subset of $R_{1}$. The functor Affin $A$ yields a subset of $R_{1}$ and is defined as follows:

(Def. 6) Affin $A=\bigcap\left\{B ; B\right.$ ranges over affine subsets of $\left.R_{1}: A \subseteq B\right\}$.

Let us consider $R_{1}$ and let $A$ be a subset of $R_{1}$. Observe that Affin $A$ is affine.

Let us consider $R_{1}$ and let $A$ be an empty subset of $R_{1}$. Note that Affin $A$ is empty.

Let us consider $R_{1}$ and let $A$ be a non empty subset of $R_{1}$. Note that Affin $A$ is non empty.

One can prove the following propositions:

(49) For every subset $A$ of $R_{1}$ holds $A \subseteq$ Affin $A$.

(50) For every affine subset $A$ of $R_{1}$ holds $A=$ Affin $A$.

(51) For all subsets $A, B$ of $R_{1}$ such that $A \subseteq B$ and $B$ is affine holds Affin $A \subseteq B$.

(52) For all subsets $A, B$ of $R_{1}$ such that $A \subseteq B$ holds Affin $A \subseteq$ Affin $B$.

(53) $\operatorname{Affin}(v+A)=v+\operatorname{Affin} A$.

(54) If $A_{1}$ is affine, then $r \cdot A_{1}$ is affine.

(55) If $r \neq 0$, then $\operatorname{Affin}\left(r \cdot A_{1}\right)=r \cdot \operatorname{Affin} A_{1}$.

(56) $\operatorname{Affin}(r \cdot A)=r \cdot \operatorname{Affin} A$.

(57) If $v \in$ Affin $A$, then Affin $A=v+\operatorname{Up}(\operatorname{Lin}(-v+A))$.

(58) $A$ is affinely independent iff for every $B$ such that $B \subseteq A$ and Affin $A=$ Affin $B$ holds $A=B$.

(59) Affin $A=\left\{\sum L ; L\right.$ ranges over linear combinations of $A$ : $\left.\operatorname{sum} L=1\right\}$.

(60) If $I \subseteq A$, then there exists an affinely independent subset $I_{1}$ of $V$ such that $I \subseteq I_{1} \subseteq A$ and Affin $I_{1}=$ Affin $A$.

(61) Let $A, B$ be finite subsets of $V$. Suppose $A$ is affinely independent and Affin $A=$ Affin $B$ and $\overline{\bar{B}} \leq \overline{\bar{A}}$. Then $B$ is affinely independent.

(62) $L$ is convex iff $\operatorname{sum} L=1$ and for every $v$ holds $0 \leq L(v)$.

(63) If $L$ is convex, then $L(x) \leq 1$.

(64) If $L$ is convex and $L(x)=1$, then the support of $L=\{x\}$.

(65) $\operatorname{conv} A \subseteq \operatorname{Affin} A$.

(66) If $x \in \operatorname{conv} A$ and $\operatorname{conv} A \backslash\{x\}$ is convex, then $x \in A$.

(67) Affin conv $A=$ Affin $A$.

(68) If conv $A \subseteq \operatorname{conv} B$, then Affin $A \subseteq$ Affin $B$.

(69) For all subsets $A, B$ of $R_{1}$ such that $A \subseteq$ Affin $B$ holds Affin $(A \cup B)=$ Affin $B$. 


\section{BARYCEntric CoORdinates}

Let us consider $V$ and let us consider $A$. Let us assume that $A$ is affinely independent. Let us consider $x$. Let us assume that $x \in$ Affin $A$. The functor $x \rightarrow A$ yielding a linear combination of $A$ is defined by:

(Def. 7$) \quad \sum(x \rightarrow A)=x$ and $\operatorname{sum} x \rightarrow A=1$.

We now state a number of propositions:

(70) If $v_{1}, v_{2} \in$ Affin $I$, then $(1-r) \cdot v_{1}+r \cdot v_{2} \rightarrow I=(1-r) \cdot\left(v_{1} \rightarrow I\right)+r \cdot\left(v_{2} \rightarrow\right.$ $I)$.

(71) If $x \in \operatorname{conv} I$, then $x \rightarrow I$ is convex and $0 \leq(x \rightarrow I)(v) \leq 1$.

(72) If $x \in \operatorname{conv} I$, then $(x \rightarrow I)(y)=1$ iff $x=y$ and $x \in I$.

(73) For every $I$ such that $x \in$ Affin $I$ and for every $v$ such that $v \in I$ holds $0 \leq(x \rightarrow I)(v)$ holds $x \in \operatorname{conv} I$.

(74) If $x \in I$, then conv $I \backslash\{x\}$ is convex.

(75) For every $B$ such that $x \in$ Affin $I$ and for every $y$ such that $y \in B$ holds $(x \rightarrow I)(y)=0$ holds $x \in \operatorname{Affin}(I \backslash B)$ and $x \rightarrow I=x \rightarrow I \backslash B$.

(76) For every $B$ such that $x \in \operatorname{conv} I$ and for every $y$ such that $y \in B$ holds $(x \rightarrow I)(y)=0$ holds $x \in \operatorname{conv} I \backslash B$.

(77) If $B \subseteq I$ and $x \in$ Affin $B$, then $x \rightarrow B=x \rightarrow I$.

(78) If $v_{1}, v_{2} \in$ Affin $A$ and $r+s=1$, then $r \cdot v_{1}+s \cdot v_{2} \in$ Affin $A$.

(79) For all finite subsets $A, B$ of $V$ such that $A$ is affinely independent and Affin $A \subseteq$ Affin $B$ holds $\overline{\bar{A}} \leq \overline{\bar{B}}$.

(80) Let $A, B$ be finite subsets of $V$. Suppose $A$ is affinely independent and Affin $A \subseteq$ Affin $B$ and $\overline{\bar{A}}=\overline{\bar{B}}$. Then $B$ is affinely independent.

(81) If $L_{10}(v) \neq L_{11}(v)$, then $\left(r \cdot L_{10}+(1-r) \cdot L_{11}\right)(v)=s$ iff $r=\frac{L_{11}(v)-s}{L_{11}(v)-L_{10}(v)}$.

(82) $A \cup\{v\}$ is affinely independent iff $A$ is affinely independent but $v \in A$ or $v \notin$ Affin $A$.

(83) If $w \notin$ Affin $A$ and $v_{1}, v_{2} \in A$ and $r \neq 1$ and $r \cdot w+(1-r) \cdot v_{1}=$ $s \cdot w+(1-s) \cdot v_{2}$, then $r=s$ and $v_{1}=v_{2}$.

(84) If $v \in I$ and $w \in \operatorname{Affin} I$ and $p \in \operatorname{Affin}(I \backslash\{v\})$ and $w=r \cdot v+(1-r) \cdot p$, then $r=(w \rightarrow I)(v)$.

\section{REFERENCES}

[1] Grzegorz Bancerek. Cardinal numbers. Formalized Mathematics, 1(2):377-382, 1990.

[2] Czesław Byliński. Binary operations applied to finite sequences. Formalized Mathematics, 1(4):643-649, 1990.

[3] Czesław Byliński. Functions and their basic properties. Formalized Mathematics, 1(1):5565,1990

[4] Czesław Byliński. Some basic properties of sets. Formalized Mathematics, 1(1):47-53, 1990. 
[5] Czesław Byliński. The sum and product of finite sequences of real numbers. Formalized Mathematics, 1(4):661-668, 1990.

[6] Noboru Endou, Takashi Mitsuishi, and Yasunari Shidama. Convex sets and convex combinations. Formalized Mathematics, 11(1):53-58, 2003.

[7] Noboru Endou, Takashi Mitsuishi, and Yasunari Shidama. Dimension of real unitary space. Formalized Mathematics, 11(1):23-28, 2003.

[8] Krzysztof Hryniewiecki. Basic properties of real numbers. Formalized Mathematics, 1(1):35-40, 1990.

[9] Jarosław Kotowicz. Real sequences and basic operations on them. Formalized Mathematics, 1(2):269-272, 1990.

[10] Andrzej Trybulec. Domains and their Cartesian products. Formalized Mathematics, 1(1):115-122, 1990.

[11] Wojciech A. Trybulec. Basis of real linear space. Formalized Mathematics, 1(5):847-850, 1990.

[12] Wojciech A. Trybulec. Linear combinations in real linear space. Formalized Mathematics, $1(3): 581-588,1990$

[13] Wojciech A. Trybulec. Vectors in real linear space. Formalized Mathematics, 1(2):291-296, 1990.

[14] Zinaida Trybulec. Properties of subsets. Formalized Mathematics, 1(1):67-71, 1990.

[15] Edmund Woronowicz. Relations and their basic properties. Formalized Mathematics, 1(1):73-83, 1990.

Received December 18, 2009 